\title{
A Neighborhood Condition for Graphs to Have Special [a,b]-Factor
}

\author{
Jinguo Lei ${ }^{1}$, Qingzhi Yu ${ }^{2}$, Changhua Huang ${ }^{1}$, Man Liu ${ }^{3}$ \\ ${ }^{1}$ Department of Aerial Four Station Support, Xuzhou Air Force Logistic College, Xuzhou, China \\ ${ }^{2}$ Department of Aerial Ammunition, Air Force Logistic College, Xuzhou, China \\ ${ }^{3}$ Department of Fundamental Courses, Air Force Logistic College, Xuzhou, China \\ Email: qz_yu@sina.com.cn
}

Received July 17, 2013; revised August 17, 2013; accepted August 25, 2013

Copyright (C) 2014 Jinguo Lei et al. This is an open access article distributed under the Creative Commons Attribution License, which permits unrestricted use, distribution, and reproduction in any medium, provided the original work is properly cited. In accordance of the Creative Commons Attribution License all Copyrights (C) 2014 are reserved for SCIRP and the owner of the intellectual property Jinguo Lei et al. All Copyright (C) 2014 are guarded by law and by SCIRP as a guardian.

\section{ABSTRACT}

Let $G$ be a graph of order $n$, and let $a$ and $b$ be integers, such that $1 \leq a<b$. Let $H$ be a subgraph of $G$ with $m(\leq b)$ edges, and $\delta(G)$ be the minimum degree. We prove that $G$ has a $[a, b]$-factor containing all edges of $H$ if $\delta(G) \geq a+m, N C(G) \geq \frac{a n+2 m}{a+b}$, and when $a \leq 2, \quad n \geq \frac{2(a+b)(a+b-1)}{b}-\frac{a+b}{b(a-1)}+\frac{2 m}{b}$.

\section{KEYWORDS}

\section{Graph; Factor; [a,b]-Factor; The Minimum Degree; Neighborhood Condition}

\section{Introduction}

We consider the finite undirected graph without loops and multiple edges. Let $G$ be a graph with vertex set $V(G)$ and edge set $E(G)$. Given $x \in V(G)$, the set of vertices adjacent to $x$ is said to be the neighborhood of $x$, denoted by $N_{G}(x) . d_{G}(x)=\left|N_{G}(x)\right|$ is called the degree of $x$ and we write $N_{G}[x]$ for $N_{G}(x) \cup\{x\}$. Furthermore we define $\delta(G)=\min \left\{d_{G}(x) \mid x \in V(G)\right\}, N C(G)=\min _{x y \notin E(G)}\left\{\left|N_{G}(x) \cup N_{G}(y)\right|\right\}$.

For a subset $S \subset V(G)$, let $G-S$ denote the subgraph obtained from $G$ by deleting all the vertices of $S$ together with the edges incident with the vertices of $S$.

Let $a$ and $b$ be integers such that $1 \leq a<b$. A $[a, b]$-factor of $G$ is defined as a spanning subgraph $F$ of $G$ such that $a \leq d_{F}(x) \leq b$ for all $x \in V(G)$. Other notations and terminology are the same as those in [1]

The existence of a factor for a graph $G$ is closely related to the degree of vertices. Concerning the minimum degree and the existence of $k$-factor Egawa, Enomoto [2] and Katerinis [3] proved that there exists $k$ factor when $n \geq 4 k-5$ and $\delta(G) \geq \frac{n}{2}$ for a graph $G$. Iida and Nishimura [4] proved that if $n \geq 4 k-5$ and $\sigma_{2}(G) \geq n$ there exists $k$-factor for a graph $G$.

H. Y. Pan [5] generalized the result of Iida and Nishimura to [a,b] -factor: if $\delta(G) \geq a, n \geq \frac{(a+b)^{2}-(a+b)}{b}$ and $\sigma_{2}(G) \geq \frac{2 a n}{a+b}, G$ has an $[a, b]$-factor.

Concerning adjacent set union and $[a, b]$-factor, in $2000 \mathrm{H}$. Matsuda gave the following result: 
Theorem 1 [5]: Let $a, b$ be integer such that $1 \leq a<b$, and $G$ be a graph of order $n$ with $n \geq \frac{2(a+b)(a+b-1)}{b}$ and $\delta(G) \geq a$.

If $\left|N_{G}(x) \cup N_{G}(y)\right| \geq \frac{a n}{a+b}$ for any two non-adjacent vertices $x$ and $y$ of $G$, then $G$ has a $[a, b]$ factor We prove the following theorem for a graph to have a $[a, b]$-factor with given properties, which is an extension of theorem 1 .

Theorem 2: Let $a$ and $b$ be integers such that $1 \leq a<b, G$ be a graph of order $n$, and $H$ be a subgraph of $G$ with $m(\leq b)$ edges. If $\delta(G) \geq a+m$, and $\left|N_{G}(x) \cup N_{G}(y)\right| \geq \frac{a n+2 m}{a+b}$ for any two non-adjacent vertices $x$ and $y$ of $G$, when $a \geq 2$, we suppose

$$
n \geq \frac{2(a+b)(a+b-1)}{b}-\frac{a+b}{b(a-1)}+\frac{2 m}{b} .
$$

Then $G$ has a $[a, b]$-factor containing all edges of $H$.

\section{Proof of Theorem 2}

Let $S$ and $T$ be two disjoint subset of $V(G), E_{1}$ and $E_{2}$ be two disjoint subset of $E(G)$. Let $W=V(G) \backslash(S \cup T)$, $E(S)=\{x y \in E(G): x, y \in S\}, E(S, T)=\{x y \mid x y \in E(G), x \in S, y \in T\}, E(T)=\{x y \in E(G): x, y \in T\}$.

$E_{1}^{\prime}=\left\{x y \in E_{1} ; x, y \in S\right\}, \quad E_{1}^{\prime \prime}=\left\{x y \in E_{1} ; x \in S, y \in V(G) \backslash(S \cup T)\right\}$

$$
E_{2}^{\prime}=\left\{x y \in E_{2}: x, y \in T\right\}, \quad E_{2}^{\prime \prime}=\left\{x y \in E_{2}: x \in T, y \in V(G) \backslash(S \cup T)\right\}
$$

$\alpha_{G}\left(S, T ; E_{1}, E_{2}\right)=2\left|E_{1}^{\prime}\right|+\left|E_{1}^{\prime}\right|, \quad \beta_{G}\left(S, T ; E_{1}, E_{2}\right)=2\left|E_{2}^{\prime}\right|+\left|E_{2}^{\prime \prime}\right|$.

Lemma 1 [6]: Let $G$ be a graph, and let $g$ and $f$ be two integer-valued functions defined on $V(G)$ such that $0 \leq g(x)<f(x) \leq d_{G}(x)$ for all $x \in V(G)$. Let $E_{1}$ and $E_{2}$ be two disjoint subsets of $E(G)$. Then $G$ has a $(g, f)$-factor $F$ such that $E_{1} \subseteq E(F)$ and $E_{2} \cap E(F)=\Phi$ if and only if for any two disjoint subsets $S$ and $T$ of $V(G)$.

$$
\delta_{G}(S, T ; g, f)=d_{G-S}(T)-g(T)+f(S) \geq \alpha_{G}\left(S, T ; E_{1}, E_{2}\right)+\beta_{G}\left(S, T ; E_{1}, E_{2}\right) .
$$

Lemma 2: Let $a$ and $b$ be integers such that $1 \leq a<b$, and $G$ be a graph, and $H$ be a subgraph of $G$. Then $G$ has a $[a, b]$-factor $F$ such that $E(H) \subseteq E(F)$ if and only if

$$
b|S|-a|T|+d_{G-S}(T) \geq \sum_{x \in S} d_{H}(x)-e_{H}(S, T) .
$$

Let $E_{1}=E(H)$ and $E_{2}=\Phi$, and we note that

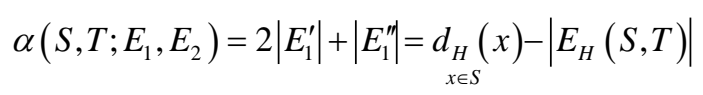

where $E_{H}(S, T)=\{x y \in E(H): x \in H, y \in T\}$ and $\beta\left(S, T ; E_{1}, E_{2}\right)=2\left|E_{2}^{\prime}\right|+\left|E_{2}^{\prime \prime}\right|=0$.

It is easy to see Lemma 2 is an immediately result of Lemma 1.

Now we prove Theorem 2: Suppose that $G$ satisfies the assumptions of Theorem 2, but it has no $[a, b]$ factor as described in Theorem 2. Then by Lemma 2 there exist two disjoint subsets $S$ and $T$ of $V(G)$ such that

$$
b|S|-a|T|+d_{G-S}(T)-\sum_{x \in S} d_{H}(x)+e_{H}(S, T)+1 \leq 0 .
$$

We choose such $S$ and $T$ so that $|T|$ is minimum. If $T=\Phi$, then by (1) we get $b|S|-\sum_{x \in S} d_{H}(x)+1 \leq 0$, 
which is a contradiction. Since $d_{H}(x) \leq m \leq b$ for all $x \in H$, hence we have $T \neq \Phi$

Suppose that there exists a vertex $\omega \in T$ such that $d_{G-S}(\omega)+e_{H}(S, \omega) \geq a$, then $S$ and $T-\{\omega\}$ satisfy (1), which contradicts the choice of $T$, therefore $d_{G-S}(x)+e_{H}(S, x) \leq a-1$ for all $x \in T$.

Now we define

$$
h_{1}=\min \left\{d_{G-S}(x)+e_{H}(S, x): x \in T\right\},
$$

and let $x_{1} \in T$ be a vertex such that

$$
h_{1}=d_{G-S}\left(x_{1}\right)+e_{H}\left(S, x_{1}\right) .
$$

Note that $h_{1} \leq a-1$ holds, we consider two cases.

Cases 1: $T=N_{T}\left[x_{1}\right]$

Note that $|S|+h_{1} \geq d_{G}\left(x_{1}\right) \geq \delta(G) \geq a+m, \sum_{x \in S} d_{H}(x) \leq 2 m, a>h_{1}$, and $b>a \geq h_{1}+1 \geq\left|N_{T}\left[x_{1}\right]\right|=|T|$.

By (1), we obtain

$$
\begin{aligned}
0 & \geq b|S|+\sum_{x \in T}\left(d_{G-S}(x)+e_{H}(S, x)\right)-a|T|-\sum_{x \in S} d_{H}(x)+1 \\
& \geq b\left(a+m-h_{1}\right)+\left(h_{1}-a\right)|T|-2 m+1 \geq\left(a-h_{1}\right)(b-|T|)+m b-2 m+1 \geq 1 .
\end{aligned}
$$

This is a contradiction.

Cases 2: $T \neq N_{T}\left[x_{1}\right]$

It is clear that $T \backslash N_{T}\left[x_{1}\right] \neq \Phi$, then we defined $h_{2}=\min \left\{d_{G-S}(x)+e_{H}(S, x): x \in T \backslash N_{T}\left[x_{1}\right]\right\}$ and let $x_{2} \in T$ be a vertex such that $h_{2}=d_{G-S}\left(x_{2}\right)+e_{H}\left(S, x_{2}\right)$ by the condition of Theorem 2, the following inequality holds:

$$
\frac{a n+2 m}{a+b} \leq\left|N_{G}\left(x_{1}\right) \cup N_{G}\left(x_{2}\right)\right| \leq|S|+h_{1}+h_{2}
$$

which implies

$$
|S| \geq \frac{a n+2 m}{a+b}-\left(h_{1}+h_{2}\right) .
$$

Note that the number of vertices in $T$ which satisfies the equality $d_{G-S}(x)+e_{H}(S, x)=h_{1}$ is at most $h_{1}+1$, and the rest of vertices in $T$ satisfy $d_{G-S}(x)+e_{H}(S, x) \geq h_{2}$.

So we obtain

$$
\sum_{x \in T}\left(d_{G-S}(x)+e_{H}(S, x)\right) \geq h_{1}\left(h_{1}+1\right)+h_{2}\left(|T|-h_{1}-1\right) .
$$

And further by (1)

$$
b|S|+h_{1}\left(h_{1}+1\right)+h_{2}\left(|T|-h_{1}-1\right)-a|T|-\sum_{x \in S} d_{H}(x)+1 \leq 0 .
$$

Note that $|S|=n-|T|-|W|$ and $\sum_{x \in S} d_{H}(x) \leq 2 m$, so we have

$$
b(n-|T|-|W|)+h_{1}\left(h_{1}+1\right)+h_{2}\left(|T|-h_{1}-1\right)-a|T|-2 m+1 \leq 0
$$

and hence

$$
|T| \geq \frac{b n+\left(h_{1}-h_{2}\right)\left(h_{1}+1\right)-2 m+1}{a+b-h_{2}}-\frac{b|W|}{b+a-h_{2}} \geq \frac{b n+\left(h_{1}-h_{2}\right)\left(h_{1}+1\right)-2 m+1}{a+b-h_{2}}-|W| .
$$

By (2) (3) we have 


$$
\begin{aligned}
n & =|S|+|T|+|W| \geq \frac{a n+2 m}{a+b}-h_{1}-h_{2}+\frac{b n+\left(h_{1}-h_{2}\right)\left(h_{1}+1\right)-2 m+1}{a+b-h_{2}}-|W|+|W| \\
& =\frac{a n+2 m}{a+b}-h_{1}-h_{2}+\frac{b n+\left(h_{1}-h_{2}\right)\left(h_{1}+1\right)-2 m+1}{a+b-h_{2}} .
\end{aligned}
$$

Let $f\left(h_{1}\right)=\frac{a n+2 m}{a+b}-h_{1}-h_{2}+\frac{b n+\left(h_{1}-h_{2}\right)\left(h_{1}+1\right)-2 m+1}{a+b-h_{2}}$.

Let $\frac{\mathrm{d} f}{\mathrm{~d} h_{1}}=0$, we have $h_{1}=\frac{a+b-1}{2} \geq a$, Note that $h_{1} \leq h_{2} \leq a-1$, it is easy to see that $f\left(h_{1}\right)$ is the minimum when $h_{1}=h_{2}$.

So we have

$$
n \geq \frac{a n+2 m}{a+b}-2 h_{2}+\frac{b n-2 m+1}{a+b-h_{2}} .
$$

If $h_{2}=0$, by (5) we have $n \geq \frac{a n+2 m}{a+b}+\frac{b n-2 m+1}{a+b}=n+\frac{1}{a+b}$.

This is a contradiction.

So we suppose $h_{2} \geq 1$, and hence $a \geq 2$. By (5) we have

$$
n \leq \frac{2(a+b)\left(a+b-h_{2}\right)}{b}-\frac{a+b}{b h_{2}}+\frac{2 m}{b} \leq \frac{2(a+b)(a+b-1)}{b}-\frac{a+b}{b(a-1)}+\frac{2 m}{b} \text {. }
$$

This is a final contradiction. Therefore theorem 2 is proved.

\section{REFERENCES}

[1] J. A. Bondy and U. S. R. Murty, “Graph Theory with Applications,” American Elsevier, New York, 1976.

[2] Y. Egawa and H. Enomoto, "Sufficient Conditions for the Existence of k-Factors," Recent Studies in Graph Theory, Vishwa International Publications, India, 1989, pp. 96-105.

[3] P. Katerinis, "Minimum Degree of a Graph and the Existence of k-Factors," Proceedings of the Indian Academy of Sciences, Vol. 94, No. 2, 1985, pp. 123-127.

[4] T. Iida and T. Nishimura, “An Ore-Type Conditions for the Existence of k-Factors in Graphs,” Graphs and Combinatorics, Vol. 7, No. 4, 1991, pp. 353-361. http://dx.doi.org/10.1007/BF01787640

[5] H. Y. Pan, “[a,b]-Facor of Graph G,” Master Paper, Shandong University, Jinan, 1996.

[6] G. Li and G. Liu, “(g,f)—Factorizations Orthogonal to a Subgraph in Graphs,” Science in China (A), Vol. 41, No. 3, 1998, pp. 267-272. http://dx.doi.org/10.1007/BF02879045 